\title{
Larreys Amputationstechnik
}

\author{
Von Beat Rüttimann
}

Auch nach bald zweihundert Jahren weiteren chirurgischen Fortschrittes sind viele berufliche Nöte und Sorgen nicht kleiner geworden; viele Probleme harren noch immer einer Lösung, und viele Irrtümer mußte man verlassen. Doch manche der damaligen Erkenntnisse dürfen auch heutigen Tages ihre Gültigkeit beanspruchen, und das verdanken wir nicht zuletzt einem großen Meister unseres Faches, der uns Sicherheit und Erfolg in der Technik, vor allem aber ein lebendiges Beispiel in mitleidender, ärztlich tätiger Menschlichkeit ließ. Aus dieser Sicht möchte ich einige Gedanken zu Larrey in seiner Zeit ${ }^{1-13}$ und zu seiner operativen Technik darlegen.

Ohne Zweifel war Larrey ein bekannter, ja berühmter Allgemein- und Feldchirurg. Und mit seinen Amputationen ganz besonders hat er sich einen weitverbreiteten Namen geschaffen, so daß in der Folge «Larrey» häufig mit «Amputation im Feld» gleichgesetzt wurde ${ }^{14,15}$. Aber eben, das allein vermag den großen Namen in Wahrheit und Wirklichkeit nicht zu begründen. Eine Amputation ${ }^{16}$ ist schließlich ein destruktiver, verstümmelnder, in mehrdeutigem Sinne heroischer Eingriff, damals und auch heute mit vielen Komplikationen und einer unsicheren Prognose behaftet. Die zweihundert in zweimal vierundzwanzig Stunden während und nach der Schlacht bei Borodino (an der Moskwa) ausgeführten Absetzungen von Gliedmaßen muten fast wahnwitzig an, wenngleich die Heilergebnisse in den Memoiren als recht gut beziffert sind.

Während der großen Napoleonischen Kriege war Larrey Chefchirurg der Kaisergarde. Niemals war er Leibchirurg Napoleons; er mußte diese brennend begehrte und beneidete Stellung Boyer ${ }^{17}$ und Yvan ${ }^{17}$ überlassen. Er war auch nicht Chefchirurg der Großen Armee, außer im unglückseligen Rußlandfeldzug. Und dennoch scheinen ihn praktisch alle Soldaten gekannt zu haben ${ }^{18}$, die zeitgenössischen Memoiren ${ }^{19-22}$ erwähnen ihn, sogar unter Umständen, da er unmöglich dabeigewesen sein kann. Er hat in der Tat an einer eindrücklichen Zahl von Gefechten und Schlachten und an allen wichtigen Feldzügen der Revolution und der Kaiserzeit teilgenommen. Napoleon nannte ihn mit der Zuwendung von 100000 Francs im zweiten Artikel an fünfzehnter Stelle seines Testamentes «l'homme le plus vertueux que j'aie connu» ${ }^{23,24}$, an anderer Stelle auch «la Providence du soldat». Das mag daher rühren, daß sich die Ambulanzen der kaiserlichen Garde nicht ausschließlich um die kranken und verletzten Gardeangehörigen kümmerten, sondern auch Patienten anderer Truppenteile, ja sogar 
des Feindes ${ }^{25,26}$, immer wieder mit unveränderter Hingabe und Aufopferung pflegten und behandelten.

Einen Bestandteil seines Rufes hat sicher ausgemacht, daß die Soldaten Larrey stets bei der Truppe - auf dem Marsch, im Biwak, während des Kampfes - und in friedlicheren Zeiten im Spital Gros Caillou und im Hôtel des Invalides sahen, so daß sie sich an seine ärztliche Gegenwart und Erscheinung gewöhnt hatten. Das wiederholt bewiesene Vertrauen des Kaisers ${ }^{27}$ befestigte ihre Hoffnungen im Falle von Verwundung oder Siechtum. Besonders dazu beigetragen hat die Erfindung, Erprobung und Einführung der «fliegenden Ambulanzen», angeregt durch frühere Ideen Percys ${ }^{28}$ und anderer, und hundertfach bewährt im völlig neuen Rhythmus, in der völlig neuen Art ${ }^{29-32}$ der Volkskriege in den Zeiten der Revolution und der Völkerschlachten während des Empire.

Beziehungen konnte Larrey erst nach und nach, und niemals in gewünschtem Maße aufbauen. Vom Kaiser hoch geehrt, von Marschällen und Generälen ${ }^{33^{-40}}$ geachtet, von den Soldaten dankbar und hoffnungsvoll verehrt, gelang es ihm doch nie, eine hohe Stufe der gesellschaftlichen Leiter zu erklimmen. Larrey war eher klein gewachsen ${ }^{41}$, kräftig und von gedrungener Statur, mit den Jahren zunehmend beleibt. Die Jugendporträts zeigen ein männlich hübsches Gesicht mit warmem und sympathischem Ausdruck. Er trug Zeit seines Lebens die Haare lang ${ }^{42}$. Ohne adelige Herkunft, dem noch nicht so hochgeschätzten Stande der Chirurgen ${ }^{43,44}$ angehörend, mit einer gründlichen, aber kaum brillant zu nennenden Ausbildung, mußte er sich Anerkennung und Autorität mühsam erarbeiten. Besonders im undankbaren Kampf mit den Behörden und speziell mit der Intendance de Guerre rieb er sich auf, ohne dieses korrupte Gesindel, wie Stendhal ${ }^{45}$ es nennt, auch nur für leiseste humanitäre Regungen zu gewinnen, und ohne den Betrügereien mit Erfolg zu steuern oder der Verschleuderung und Versetzung des so dringend benötigten Sanitätsmaterials Einhalt gebieten zu können. Diese Bemühungen blieben zwar der Truppe weitgehend unbekannt; dennoch wurden kaum jemals Zweifel am guten Willen und an der Ehrlichkeit Larreys selber laut.

Die operative Technik umfaßt nicht nur das handwerkliche Können und Beherrschen des Eingriffes, sondern mit ebensolchem Gewicht die richtige Indikationsstellung, die Planung der Behandlung, die Vorbereitung des Patienten und der Operation, die Nachsorge und im Falle einer Amputation die Abgabe von Behelfen und Prothesen. Hier finden wir konkrete Hinweise für die wahre Persönlichkeit Larreys und für seine menschliche und ärztliche Größe.

Seine Indikationen waren äußerst mutig und dezidiert, und sie blieben auch nicht unangefochten oder zumindest angezweifelt und diskutiert von seinem Lehrmeister Sabatier ${ }^{46}$ und seinen Kollegen, wie Percy, der viel zurückhaltender 
war, oder Heinrich von Roos ${ }^{47}$ und Turiot ${ }^{48}$, die überhaupt nicht gern amputierten. Larrey befürwortete das Absetzen einer Gliedmaße immer dann, wenn eine schwere Zerstörung einer Extremität vorlag, wenn sich nicht anderweitig beherrschbare Hämorrhagien einstellten, wenn müde Geschosse ausgedehnte Kontusionsverletzungen hervorgerufen hatten, wenn große Gelenke breit eröffnet waren, wenn Fremdkörper nicht extrahiert werden konnten, bei vollständiger Läsion des Bandapparates großer Gelenke, bei Tetanus und Gasbrand und bei «Fièvre catarrhale de congélation».

Im Gegensatz zur damaligen Schulmeinung, besonders zu der von der Académie prämierten Arbeit von Faure wie auch zu den Ansichten von Percy, Yvan und Dubois ${ }^{17}$, setzte Larrey den Zeitpunkt einer als notwendig erachteten Amputation so früh wie möglich an, wenn irgendwie durchführbar vor dem Auftreten der «accidens primitifs » oder «premiers accidens». Damit berücksichtigte er - wie vor ihm schon Paré ${ }^{49}$ - die in ihrer Bedeutung bereits erkannten psychologischen Momente. Dem großen Einfluß schwerer Verletzungen auf das ganze Nervensystem, auf die "vie organique» und die «vie interne», trug er durch die frühe Amputation oder Exartikulation Rechnung, indem er saubere und ans Gesunde grenzende Wunden ${ }^{50}$ schuf, ohne Taschen, ohne Sickerblutungen, ohne Fremdkörper oder Knochenfragmente. Wollte man in dieser Weise vorgehen, so mußte der Eingriff seiner Ansicht nach sofort und in jedem Fall innerhalb der 24-StundenGrenze erfolgen. Er ist nicht der erste, der diese Ideen äußerte, aber er hat sie ausgebaut, begründet, verfochten und weit verbreitet.

Zur Schmerzbekämpfung: Durch den früh angesetzten Operationszeitpunkt konnten Verletzungsschock und hämorrhagischer Schockzustand voll ausgenützt werden. Bei großer Kälte ist die Schmerzempfindung ebenfalls betäubt. Solange es vorrätig war, enthielten die Sanitätskisten Laudanum; sonst mußten Alkoholika den gleichen Dienst tun, so der von Larrey immer wieder genannte «chenaps». Er legte auch großen Wert auf die Ermutigung, die Verletzte erfuhren, wenn sie sahen, daß bereits operierte Kameraden nicht mehr unter unsäglichen Schmerzen litten und zu schreien aufhörten, wenn der Eingriff beendet war. Unmittelbar nach der Verwundung mochten auch die «exaltation des esprits» und «le cœur gonflé d'Honneur» (nach Guillemeau) im Sinne einer Analgesie nachwirken ${ }^{51}$. Daneben spielte sicher die Individualität der Schmerzempfindung (nach Leriche ${ }^{52}$ ) eine Rolle, und vielleicht waren diese Menschen Unbill und Beschwerden oft tatsächlich weniger zugänglich.

Zur Technik der einzelnen ablativen Eingriffe $e^{1,9,46}$ hat Larrey keine bahnbrechenden, grundlegenden Neuerungen gebracht; hingegen konnte er sie bis ins letzte ausfeilen und sie derart vereinfachen und trainieren, daß sie auch von 
weniger geübten und unerfahrenen Chirurgen gefahrlos und mit einiger Aussicht auf Erfolg genutzt werden konnte. Dies gilt vor allem für sein Verfahren der Exartikulation der oberen Extremität im Schultergelenk. Er hat diese der Oberarmamputation vorgezogen und sich damit in Gegensatz zu vielen seiner Kollegen und zu den englischen Chirurgen gesetzt. Nach seiner Auffassung sprachen gegen die Absetzung im Oberarm folgende Gründe: Bei kurzen Stümpfen und gleichzeitig verletztem Musculus deltoideus ziehen die Musculi pectoralis major und latissimus dorsi die Weichteile über den knöchernen Stumpf zurück. Die Ligaturen der Gefäße gestalten sich sehr schwierig, und die Gefahr einer Mitnahme von Anteilen des Plexus brachialis ist groß (und Nervenligaturen lösen nach damaliger Ansicht Starrkrampf aus). Das Schultergelenk ankylosiert in der Regel, so daß der Stumpf dann störend wirkt und die prothetische Versorgung erschwert (was nicht zutrifft für Nelson, der eine gute Restbeweglichkeit besaß und keine Prothese trug!). Die Durchtrennung des Humerus vergrößert nur die Wundfläche und verlängert die Dauer des Eingriffes. Nachdem sich die zuerst geübte Methode als technisch zu anspruchsvoll erwiesen hatte, gab Larrey später ein anderes Vorgehen an. Er distanzierte sich damit etwas von den Lehrmeinungen Desaults und Lafayes, erzielte aber sehr erfreuliche Resultate. Zunächst nach einer vertikalen Inzision über den Musculus deltoideus geschah die Bildung eines posterioren, dann vom selben Schnitt aus eines anterioren Lappens, die Durchtrennung der Schultergelenkkapsel und der dortigen Sehnen mit einem KnopfBistouri, die Luxation des Humeruskopfes, manuelle Gefäßkompression (unter Sicht!) durch einen Operationsgehilfen, schließlich die Durchtrennung der axillär verbliebenen Brücke. Nach Setzen der Ligaturen Adaptation der Wundränder ohne Nähte ausschließlich durch einen speziellen, aber einfach anzulegenden Verband. Auf diese Weise war auch die allenfalls notwendige gleichzeitige Entfernung abgesprengter Fragmente der angrenzenden ossären Strukturen möglich, ebenso die weitere Darstellung und Ligatur einer verletzten Arteria subclavia. Der Heilverlauf an Nelsons Oberarmstumpf ${ }^{53,54}$ als Einzelfall scheint Larreys Überlegungen und besonders seinen Befürchtungen recht zu geben, ebenfalls als Einzelfall die erfolgreiche Schulterexartikulation des Artillerieobersten d'Aboville, der eine schwere, die ganze Schulterregion destruierende Verletzung erlitten hatte und nach ungestörtem Verlauf in ansprechender Art prothetisch versorgt werden konnte (Musée de l'Armée).

Die koxofemorale Exartikulation war angezeigt bei einer hohen traumatischen Oberschenkelamputation, einer hohen Femurfraktur mit Läsion der Gefäße und Nerven und einer hochreichenden Kontusionsverletzung des Oberschenkels mit «désorganisation interne». Die Vorteile dieser Exartikulation gegenüber der 
hohen Oberschenkelamputation umfaßten nach Larreys Ansicht wiederum die wegfallende Notwendigkeit einer Knochendurchtrennung und die geringere Masse der durchzuschneidenden Muskulatur. Technik des Eingriffes : Leistenschnitt mit Darstellung der Gefäße, Massenligatur mit Hilfe der von Larrey selber vorgeschlagenen und in seinen Memoiren auch abgebildeten «aiguille courbe mousse». Bildung des inneren medialen Lappens durch Schnittführung oberhalb des Trochanter minor nach schräg unten und innen. Ligatur blutender Gefäße. Eröffnung der Hüftgelenkskapsel, Abduktion der Extremität durch einen Gehilfen, breite Eröffnung des Gelenkes, Luxation des Femurkopfes und Durchtrennung des Ligamentum capitis femoris. Bildung des äußeren Lappens durch Schnittführung oberhalb des Trochanter major nach schräg unten und außen. Kontrolle der Hämostase mit Ligatur aller noch blutenden Gefäße. Adaptation der beiden Lappen durch einige Einzelknopfnähte mit der weniger biegsamen Rundnadel von Larrey, jedoch nur Haut und Subcutis fassend. Gekreuzter Longuettenverband und Anlegen einer einfachen Bandage.

Dieser Eingriff wurde auch von Larrey nicht allzu häufig ausgeführt, und die rapportierten Ergebnisse einiger weniger Fälle sind nicht gerade ermutigend, wenngleich die Patienten nach seiner Ansicht an verschiedenen Ursachen wie hinzukommenden Zweiterkrankungen und nicht an postoperativen Komplikationen verstarben. Larrey versprach sich von diesem Eingriff Erfolge, indem Patienten mit schwersten Beinverletzungen unmittelbar geholfen werden konnte, man nicht rein abwartend bleiben mußte und es auch gelang, eine Linderung der schlimmsten Schmerzen zu erzielen. Eine Anzeige für diese große Operation sah er auch in weiteren psychologischen Gründen, so in der Rücksichtnahme auf die Moral der Truppe und anderer Verletzter.

Weitere Absetzungsoperationen an den unteren Extremitäten waren die häufigen Ober- und Unterschenkelamputationen. Das große Problem war die Neigung der Weichteile, sich über den knöchernen Stumpf zu retrahieren. Solche Amputationsstümpfe wurden dann konisch, sehr empfindlich, schwierig zu versorgen und wiesen eine große Neigung zu rezidivierenden Hautdefekten und zu wiederholt angehenden Infektionen auf. Um dieser Gefahr auszuweichen, benutzte Percy bei der Operation einen Weichteil-Retraktor, ein eigens zu diesem Zweck gefertigtes Instrument, das erlaubte, die Weichteile über den durchzusägenden Knochenschaft zurückzuziehen und die knöcherne Absetzung auf höherem Niveau als die Weichteildurchtrennung vorzunehmen.

Für die Amputation im Unterschenkel folgte Larrey der Meinung von Petit: Zirkuläre Durchtrennung von Haut und Subcutis, Zurückschieben dieser Weichteilschicht, Durchtrennung der oberflächlichen Muskulatur, Darstellung und 
Ligatur (Massenligatur) der Gefäße, Durchtrennung der Nerven, weiteres Zurückschieben der bereits durchschnittenen Weichteile, Resektion der tiefen Muskelschicht und schließlich noch weiter proximal Durchsägen des Knochens. Bei hoher Amputation gleichzeitige Resektion und Exstirpation des Fibulaköpfchens. Prinzipiell keine Lappenbildung (im Gegensatz beispielsweise zu Verduyn, der 1695 eine Z-förmige Schnittführung mit Bildung eines großen hinteren Lappens angab, und auch zu den heutigen Ansichten, wobei allerdings Fälle mit schwerem Infekt und die Lehrmeinungen der aktuellen Feldchirurgie ausgeklammert seien). Die Ligaturen wurden lang belassen und fielen ab dem fünften Tag, in der Regel zwischen dem achten und zehnten postoperativen Tag ab. Die Adaptation der Weichteile geschah ohne Nähte, nur mit «bandelettes agglutinatives» und mit der Bandage. Auf die Wunde kam gefenstertes Leinen zu liegen, das vorher in warmen, mit Zucker oder Honig versetzten Wein getaucht wurde. Der ganze Eingriff dauerte etwa zwei Minuten.

Ähnlich war das Vorgehen bei der Amputation im Oberschenkel; doch bei sehr hohem Niveau wurden ausnahmsweise zwei Lappen gebildet, ähnlich wie bei der Hüftexartikulation, jedoch kleiner und ohne adaptierende Einzelknopfnähte. Eine zusätzliche Indikation zur Vornahme dieses Eingriffes stellten distale Femurfrakturen dar, wenn gleichzeitig rechtwinklig dazu ein transkondylärer Bruch verlief.

Weiter distal findet eigentlich nur noch die Vorfußamputation gesonderte Erwähnung, entweder in der Technik nach Villermé/Lisfranc, oder aber etwas weiter proximal nach Chopart. Die Exartikulation im Ellbogengelenk war verpönt; die Absetzung im Vorderarm und die Absetzungen der Hand, einzelner Finger oder Zehen waren offenbar ohne technische Schwierigkeiten und derart geläufig und viel geübt, daß Larrey und andere Autoren kaum Worte darüber verlieren ${ }^{55}$.

Larrey selber muß ein hervorragender Operateur gewesen sein, Vertrauen einflößend, sicher, mit ruhiger Hand und einer enormen Schnelligkeit. Innerhalb nur weniger Minuten war ein Eingriff beendet, und die fast nicht glaubhaften Operationszeiten ${ }^{56}$ von 15 Sekunden (allerdings ohne Ligatur) bis maximal 4 Minuten finden auch außerhalb seiner Erinnerungen mehrfache Bestätigung. Beim Fehlen einer zuverlässigen Analgesie oder Anästhesie war die Gewandtheit und Raschheit natürlich das hervorstechendste Merkmal eines guten Chirurgen.

Auch der postoperativen Betreuung, der Nachbehandlung und der allenfalls notwendigen prothetischen Versorgung widmete Larrey große Aufmerksamkeit. Er beobachtete und beschrieb den postoperativen Verlauf ganz genau: Nach einigen, meist nach drei Tagen beginnt sich die «suppuration» gutartigen Charakters (pus bonum et laudabile) einzustellen. Die ersten Nächte können «orageux» sein; Beruhigung folgt spätestens nach einer Woche, es sei denn, der Verlauf ge- 
stalte sich ungünstig. Vielfältige, meist symptomatische äußerliche und innerliche Heilmittel wurden verwendet. Möglichst später und seltener Verbandwechsel, rasche Mobilisation des Patienten, kräftige Nahrung und geschickte psychologische Führung ergaben einen Maßstab für gute Resultate, der für die kommenden Jahrzehnte ${ }^{57}$ Gültigkeit behalten durfte. Wobei allerdings nicht verschwiegen sei, daß auch Larrey bei der Mitteilung seiner Behandlungserfolge etwas geschwindelt hat, wie viele große Herren der damaligen medizinischen Szene ${ }^{58}$.

Die prothetische Versorgung umfaßte für den Unterschenkelamputierten die Anfertigung eines Holzköchers mit Stelzbein und mit Lederriemenbefestigung. Der Patient mußte für diesen Behelf selber aufkommen und auch darum bemüht sein. Bei hoher Unterschenkelamputation - und Larrey pflegte ja auch, wenn immer möglich, drei Querfinger unterhalb der Tuberositas tibiae oder sogar noch höher bis zum eigentlichen Tuberositasbereich, jedoch unter Belassung des Kniegelenk-Bandapparates und besonders des Ligamentum patellae abzusetzen - war die Möglichkeit gegeben, das Knie in Rechtwinkelstellung einzubetten, was von Larrey als großer Vorteil gewertet wurde. Wohlhabende Patienten ließen sich prothetische Behelfe aus Metall anfertigen, die anatomisch geformt und mit beweglichem Knie- und Sprunggelenk versehen waren. Doch auch sie verwendeten für den Alltag gewöhnlich ein Stelzbein, vielleicht mit einem zusätzlichen, feststellbaren Kniescharnier, wie man es von der Prothese des Generals Daumesnil weiß. Die Schulterkappe von d'Aboville wurde bereits erwähnt.

Viele andere Leistungen Larreys in allen anderen Gebieten der Chirurgie müssen unerwähnt bleiben. Es sei einzig noch auf seine unablässige und eindringliche Ermunterung zur Vornahme des Wund-Débridements hingewiesen, auf seinen Grundsatz des «rendre la plaie simple». Diese Forderung hat bis auf den heutigen Tag nichts von ihrer Wichtigkeit eingebüßt.

Was hat nun aus dem vielbeschäftigten Chefchirurgen der kaiserlichen Garde den berühmten Larrey gemacht? Wie erhob er sich über das allgemein noch tiefe Niveau der chirurgischen Technik und des beruflichen Ethos? Anhaltspunkte für eine Antwort ergeben sich weniger in seinen Erfindungen, in seinen fachlichen Neuerungen, in der Publikation seiner Erinnerungen und Lehrmeinungen, als in der Art, wie er die Heilkunde eben nicht als Technik, sondern als eine Kunst und kunstvoll übte. Als Mensch muß er sich den Beinamen der «Providence» verdient haben als zuverlässiger Kamerad und treuer Helfer, erfüllt von den Idealen der Revolutionszeit, der Menschlichkeit und der reinsten ärztlichen Berufung. Dafür finden sich - verstreut im Schrifttum des Zeitabschnittes - Zeugnisse, und sie bestätigen bei genauerem Zusehen nicht nur den bekannten Amputeur, sondern ebenso den großen Arzt und seine Kunst des Heilens. 


\section{Bibliographie und Anmerkungen}

${ }^{1}$ Larrey, Dominique Jean, Mémoires de Chirurgie Militaire et Campagnes. Tome I-III, Paris: J.Smith et F. Buisson 1812 (Eigenbesitz).

Larrey, Dominique Jean, Mémoires de Chirurgie Militaire et Campagnes. Tome IV, Paris: J.Smith 1817. Dieser Band (wie übrigens auch die Bände I-III) u. a. in der Eidg. Militärbibliothek Bern.

Im Zusammenhang mit der Amputationstechnik wichtigste Textstellen:

Band I: $\quad$ S. 70, 76, 91f., 272.

Band II: S. 166f., 170f., 180f. (Hüftexartikulation: Indikation und Technik), $451 \mathrm{f}$. (Mémoire sur les amputations).

Band III: S. 51f., 349f., 354f. (d'Aboville), 372f., 378f. (Unterschenkelamputation), $389 \mathrm{f}$.

Band IV: S. 27, 50, 427.

Larrey, Dominique Jean, Chirurgische Klinik oder Ergebnisse der von ihm, vorzüglich im Felde und in den Militärlazaretten, seit 1792 bis 1829 gesammelten wundärztlichen Erfahrungen. Aus dem Französischen übersetzt und mit Anmerkungen versehen von Dr. Albert Sachs. Berlin: Verlag der Buchhandlung von C.Fr. Amelang 1831.

Die benutzte Ausgabe trägt den Namenszug Dr. Krönleins und steht in der Universitätsspital-Bibliothek Zürich.

${ }^{2}$ Soubiran, André, Le Baron Larrey. Chirurgien de Napoléon. Paris: Librairie Arthème Fayard 1966.

${ }^{3}$ Richardson, Robert G., Larrey: Surgeon to Napoleon's Imperial Guard, London: John Murray (Publishers) Ltd. 1974.

${ }^{4}$ Horndasch, Max, Der Chirurg Napoleons. Das Leben des Jean-Dominique Larrey. Bonn: Karl Glöckner Verlag 1948.

${ }^{5}$ Bérenger Féraud, L.-J.-B., Le Baron Hippolyte Larrey, Paris: Librairie Fayard Frères 1899. Biographie des Sohnes von Dominique Jean Larrey, in den ersten Abschnitten mit ausgiebiger Wiedergabe der Verhältnisse und des Briefwechsels in der Familie, mit vielen Hinweisen auf das Leben und das Wirken des Vaters, besonders in seinen späteren Jahren, und mit der Schilderung seiner letzten Reise, seiner Krankheit und seines Todes.

${ }^{6}$ Huard, Pierre, Sciences - Médecine - Pharmacie de la Révolution à l'Empire (1789-1815), Paris: Les Editions Roger Dacosta 1970.

- Le Service de Santé Militaire (S. 41f.).

- Logistique et tactique sanitaires (S. $53 \mathrm{f}$.).

- Blessures de guerre (S. $55 \mathrm{f}$.).

Amputations (S. 63f.).

Prothèse (S. 75).

- Le personnel du Service de Santé (S. 81f.).

- La vie professionnelle [des médecins] (S. 277f.).

- Le statut social du médecin (S. 283f.).

${ }^{7}$ de Fourmestraux, Jacques, Larrey 1766-1842, in: Les Médecins célèbres, Genève: Editions d'Art Lucien Mazenod 1947.

${ }^{8}$ Dumesnil, René, Histoire illustrée de la Médecine, Paris: Editions d'Histoire et d'Art, Librairie Plon, 1935. 
${ }^{9}$ Richerand, M. le Chevalier, Histoire des Progrès récens de la Chirurgie, Paris: Béchet jeune, Librairie de l'Académie Royale de Médecine, 1825.

Amputations des membres: S. 175-204. Hinweise auf die grundlegenden Vorarbeiten von Paré und Petit.

- Schulterexartikulation (Technik nach Ledran père, Lafaye, Dupuytren, Champesme, Lysfranc [sic]. Larrey nicht erwähnt).

- Ellbogenexartikulation (à blâmer).

- Hüftexartikulation (Technik nach Larrey und Guthrie).

- Knieexartikulation (doit être abandonnée. Die Technik nach Fabry von Hilden, Hoin und Petit wird diskutiert).

- Vorfußamputation (Technik nach Chopart um 1793, Richerands eigene Technik, Vorgehen von Garengeot und Percy, dann von Lysfranc. Villermé nicht erwähnt).

- Unterschenkelamputation (Empfehlung, den sofortigen Wundverschluß anzustreben und die Schnittränder leicht schräg, von innen nach außen, zu vereinen. Vorschlag Béclards, die Tibia-Kante abzurunden).

- Amputation des ersten Metakarpale-Knochens nach Boyer und Béclard.

Die sofortige Vereinigung der Wundränder nannte Richerand «la méthode anglaise»; die Drainage der so vernähten Wunde erfolgte durch den einen, lang belassenen Schenkel der zur Hämostase gesetzten Ligaturen. Auf diese Weise glaubte er am besten der «rétraction consécutive» der Weichteile steuern zu können. Larrey hat dieses Vorgehen nie gebilligt und geübt, und zumindest im Falle von Kriegsverletzungen und von infizierten Amputationsstümpfen hat er bis auf den heutigen Tag in den allermeisten Fällen recht behalten. Die primäre Naht in der Feldchirurgie hat im 19. und 20. Jahrhundert unzählige Opfer gefordert, und sie wurde leider häufig durchgeführt, obwohl immer wieder einsame Mahner dagegen auftraten.

${ }^{10}$ Ackerknecht, Erwin H., Pariser Chirurgie von 1794 bis 1850. In: Gesnerus 17 (1960), S. 137-144.

11 Chevalier, A.G., Probleme des Sanitätswesens in den Napoleonischen Heeren. In: Ciba Zeitschrift Nummer 75 (1940), S. 2582-2588.

12 Richardson, Hubert N.B., A Dictionary of Napoleon and His Times, New York: Funk and Wagnalls Company o. J.

Unter dem Stichwort Larrey eine kurze, wohlausgewogene Beschreibung seines Lebenslaufes, seines Werkes, seiner Hingabe und Tapferkeit.

${ }^{13}$ Dupuy, Ernest R., and Dupuy, Trevor N., The Encyclopedia of Military History, London: Macdonald and Jane's, Revised Edition 1977.

14 Eiselsberg, Anton Freiherr von, Lebensweg eines Chirurgen, Innsbruck/Wien: TyroliaVerlag 1949.

Bei der Schilderung einer von Billroth besonders schnell und geschickt durchgeführten Schulterexartikulation erinnert der Autor an die diesbezügliche Meisterschaft Larreys (Abschnitt: Promotion und Operationszögling bei Billroth).

${ }^{15}$ Sauerbruch, Ferdinand, Das war mein Leben, Bad Wörishofen: Kindler und Schiermeyer Verlag 1951.

Bei aller Zurückhaltung in der Beurteilung dieser Autobiographie finden sich auf den Seiten 228f. und 242f. zwei Passagen, die einerseits auf die Leistungen Larreys in der feldmäßigen Amputationschirurgie eingehen, andererseits aber auch seine ärztliche Per- 
sönlichkeit eindringlich und überzeugend darstellen. Dieser Eindruck und diese Empfindungen bei der Lektüre von Larreys Schriften wurden durch die fortschreitende Zerebralsklerose kaum verfälscht; sie wirken ehrlich erlebt und verraten aufrichtige Bewunderung für diesen Chirurgen, den er als eine epochale Erscheinung würdigt.

${ }^{16}$ Im Zusammenhang mit der Exartikulation im Hüftgelenk schreibt Larrey in seinen Memoiren (Anm. 1), Band II, S. 181: "Quelque cruelle que puisse être une opération, elle est un acte d'humanité entre les mains du chirurgien, dès qu'elle peut sauver les jours d'un blessé, qui sont en danger; et plus le danger est grand et pressant, plus les secours doivent être prompts et énergiques. Ad extremos morbos, extrema remedia exquisitè optima (Hippocr.). Dans cette circonstance, l'homme de l'art fait son devoir, et ne songe point à sa réputation.»

${ }^{17}$ Chevalier, A.G., Die kaiserlichen Leibärzte. In: Ciba Zeitschrift Nummer 75 (1940), S. 2578-2581. Siehe auch Huard (Anm. 6), Ackerknecht (Anm. 10 und 58).

${ }^{18}$ Gerade in diesem Rußlandfeldzug durfte Larrey die schönsten Beweise seiner Beliebtheit und seiner Verehrung bei der Truppe erfahren. Aus seinen Memoiren (Anm. 1) und aus einem Brief an seine Gattin (zitiert bei Soubiran, Anm. 2) geht hervor, daß die Soldaten ihn im verzweifelten Gedränge vor der noch nicht eingebrochenen Brücke über die Beresina erkannten und von Soldat zu Soldat, von Hand zu Hand, ans jenseitige Ufer brachten. Sie gaben ihm Nahrung, boten ihm Schutz gegen die Kosaken, hoben ihn vom Schnee auf, stützten ihn und ließen ihn an ihr Feuer treten, während viele Generäle und hohe Offiziere zurückgewiesen wurden. Diese Soldaten dankten Larrey für seine menschliche und ärztliche Leistung, wie er ihnen sein Überleben in Rußland verdankt.

${ }^{19}$ Coignet, Memoiren des Capitaine Coignet. Von Marengo bis Waterloo, Stuttgart: Verlag von Robert Lutz o. J.

Napoleon hat Coignet als erstem das Kreuz der Ehrenlegion an die Brust geheftet, unmittelbar nach der Stiftung dieses Ordens.

${ }^{20}$ Bourgogne, François, Kriegserlebnisse 1812, Stuttgart: Verlag von Robert Lutz o. J. Seite 26 erzählt Dumont, ein Landsmann von Bourgogne, nach der Schlacht an der Moskwa folgende Episode: «Ein Chirurg des kaiserlichen Stabes, M. Larrey, welcher hier anwesend war, nahm ihr [der Marketenderin Florencia] sogleich den Daumen ab, und mir die Kugel aus dem Arm, worauf ich mich bald viel besser befand.»

${ }^{21}$ Parquin, Denis-Charles, Souvenirs de Gloire et d'Amour, Paris: Librairie Illustrée, Jules Tallandier, Editeur 1911.

Parquin selber wurde zwölfmal verwundet; er berichtet auch über zahlreiche Verletzungen seiner Gegner und seiner Kameraden. Als Angehöriger der Jungen und der Alten Garde hatte er verschiedentlich Gelegenheit, die Dienste von Larreys Ambulanz zu beanspruchen.

22 Marbot, Marcellin, Memoiren des französischen Generals Marcellin de Marbot, Stuttgart: Verlag von Robert Lutz 1899.

Marbot nennt verschiedene Ärzte namentlich. Von Larrey ist ausführlich die Rede im Zusammenhang mit der Verwundung, der operativen Behandlung und dem Tod des Marschalls Lannes (Band II). Marbot war selber dreizehnmal verwundet.

${ }^{23}$ Gallois, Léonard, Histoire de Napoléon d'après lui-même, suivie de son Testament original, Paris: Mme V ${ }^{\mathrm{e}}$ Charles-Béchet, Editeur 1829. 
${ }^{24}$ Las Cases, Comte de, Mémoires de Napoléon I ${ }^{\text {er }}$ (Le Mémorial de Ste-Hélène), Tome XVII, Paris: Cocuaud \& Cie, Editeurs o. J.

${ }^{25}$ Blanco, Richard L., Wellington's Surgeon General: Sir James McGrigor, Durham, N. C.: Duke University Press 1974.

Larrey und Mc Grigor hatten sich bereits in Ägypten und dann nach dem Waffenstillstand von 1814 in Paris getroffen. Dort fand auch eine Begegnung mit den Militärärzten Baron Pierre-François Percy und Baron René-Nicholas Desgenettes statt. Blanco zitiert auch einen Londoner Rechtsanwalt, James Simpson, der bemerkte: «Something, if possible, beyond the average care for the sick and hurt, appeared to me to animate all the medical men for the wounded of Waterloo; and their zeal made no distinction on wether their countrymen or the ennemy. » $\mathrm{Zu}$ dieser Zeit waren viele französische Militärärzte in Brüssel, unter ihnen auch Larrey nach seiner Gefangennahme durch die Preußen.

Als «the English Larrey » galt George James Guthrie, der sich ebenfalls in der Amputationschirurgie einen besonderen Namen machte. Zehn Jahre nach dem Krieg kennt auch das medizinische Frankreich seine Leistungen (siehe Anm. 9).

${ }^{26}$ Parkinson, Roger, Blücher. Der «Marschall Vorwärts», München, List Verlag 1976.

Blüchers ältester Sohn Franz war bei Teplitz durch einen Hieb am Kopf verletzt worden. Napoleon selber nahm sich seiner an, und Larrey leitete - wie er selber in den Memoiren berichtet - seine Behandlung. Blücher zeigte sich nach der preußischen Gefangennahme Larrey gegenüber erkenntlich und behandelte ihn großzügig.

${ }^{27}$ Die wichtigsten Gunstbeweise Napoleons seien hier kurz aufgeführt: Nach der (Land-) Schlacht bei Aboukir erhielt Larrey eine edle Damaszenerklinge mit Gravur, 1801 eine Dose mit dem Porträt des Ersten Konsuls und ein Geldgeschenk; am 16. August 1804 wurde er zum Offizier der Ehrenlegion ernannt; am Tage nach der Schlacht von Eylau gab ihm der Kaiser seinen eigenen Degen; 1807 wurde er Chevalier de l'Ordre de la Couronne de Fer, 1808 Commandant de la Légion d'Honneur. Im gleichen Jahr folgten Baronie des Kaiserreiches, eine Dotation in Schwedisch-Pommern und eine Rente. 1813 übergab ihm Napoleon eine diamantenbesetzte Miniatur, ein Geldgeschenk in Goldfranken und eine Staatsrente.

${ }^{28}$ Baron Pierre-François Percy (1754-1825). Berühmter Kollege und zeitweiliger Rivale Larreys, wie dieser ein hervorragender Chirurg. Chirurgien en chef de la Grande Armée (mit Ausnahme des Rußlandfeldzuges). Er machte sich ebenfalls um den (Wieder-) Aufbau des Sanitätsdienstes verdient und sorgte für die Einführung mobiler chirurgischer Sanitätsequipen auf entsprechend zugerüsteten Artilleriezubehör- und -munitionswagen («wurst»). Weniger bekannt und volkstümlich als Larrey, stand er ihm in wissenschaftlichen Belangen jedoch kaum nach. (Siehe Anm. 1, 2, 3, 6, 10, 11, 17, 25 und 58.)

${ }^{29}$ Rothenberg, Gunther E., The Art of Warfare in the Age of Napoleon, London: B.T.Batsford 1977.

Medical services (7.Kapitel, S. 225 f.): French medical services 1792-1795 (S. 227f.), Austrian, Prussian, Russian and British medical services (S. 231f.); The practice of surgery on the battlefield (S. 234f.); The French Revolutionary Armies und their New Art of War (4. Kapitel, S. $95 \mathrm{f}$.).

${ }^{30}$ Hughes, B.P., La Puissance de Feu. L'efficacité des armes à feu sur les champs de bataille, 1630-1850, Lausanne: Edita S.A. 1976. 
${ }^{31}$ Yorck v. Wartenburg, Graf, Napoleon als Feldherr, Berlin: Ernst Siegfried Mittler und Sohn, 5.Auflage 1909.

${ }^{32}$ Napoleon I., Militärische Schriften. Erläutert durch Boie. Berlin: F.Schneider \& Co. 1881.

${ }^{33}$ Chardigny, Louis, Les maréchaux de Napoléon, Paris: Librairie Jules Tallandier 1977. Besonders mit den Kommandanten der Konsular- und der Kaisergarde pflegte Larrey engere, teilweise nicht nur dienstliche Beziehungen. Unter anderen empfing er als Gäste bei sich zu Hause Eugène de Beauharnais, Murat, Lannes und Duroc.

${ }^{34}$ Gavard, Ch., Galerie des Maréchaux de France, Paris: Bureau des Galeries historiques de Versailles 1839.

Die Namen der in Anm. 33 genannten Marschälle gehen auch aus den hier wiedergegebenen Dienst-Etats hervor.

${ }^{35}$ Dunn-Pattison, R.P., Napoleon's Marshals. First published 1909. East Ardsley, Wakefield, Yorkshire: EP Publishing Limited 1977.

Die Verwundungen Lannes' bei Aboukir, Saint-Jean-d'Acre, Burgos und auf der Insel Lobau wurden alle von Larrey behandelt.

${ }^{36}$ Macdonell, A.G., Napoleon und seine Marschälle, Leipzig und Wien: E.P.Tal \& Co. Verlag 1936.

Person und Tätigkeit des Generalarztes Larrey sind nahtlos eingefügt in die farbige Schilderung des Napoleonischen Epos. Die einzelnen Charaktere der Marschälle sind plastisch ausgearbeitet und erlauben, die Art ihrer Beziehungen zu Larrey kennenzulernen, soweit sie nicht schon historische Tatsache oder zumindest geschichtliche Anekdote sind. Beispielsweise hören wir vom freundschaftlichen Verhältnis unter den Südwestfranzosen (Larrey, Murat, Lannes, Bessières).

${ }^{37}$ Six, Georges, Dictionnaire biographique des Généraux et Amiraux Français (1792-1814), Paris: G.Saffroy 1934.

Alle Verwundungen der aufgeführten Offiziere sind sorgfältig festgehalten, jedoch in der Regel ohne Nennung des behandelnden Arztes.

${ }^{38}$ Lucas-Dubreton, J., Le Maréchal Ney, Paris: Librairie Arthème Fayard o. J.

Die Bezeichnung Napoleons «le brave des braves» darf nur Ney zu Recht beanspruchen. Sie wird auch im Zusammenhang mit Larrey angeführt (siehe Anm. 14), aber in Verwechslung des Zitates. Von Larrey hat Napoleon gesagt: "Quel homme, quel brave et digne homme que Larrey!» (siehe Anm. 2).

${ }^{39}$ Lucas-Dubreton, J., Murat, Paris: Librairie Arthème Fayard 1944.

Murat war in Madrid heftig erkrankt, und es bestand der Verdacht einer Vergiftung. Entgegen dieser Annahme stellte Larrey die Diagnose einer «colique de Madrid» und behandelte ihn entsprechend seiner Lehrmeinung mit Erfolg (siehe Anm. 1, Band III, S. 134, 170f. (Mémoire sur la colique de Madrid) und S. 205 mit der Verlaufsbeschreibung der Krankheit Murats).

${ }^{40}$ Es würde hier zu weit führen, die Beziehungen Larreys zu den anderen Marschällen und Generälen des Kaiserreiches darzustellen. Zudem sind die Hinweise in Memoiren und Biographien spärlich (Macdonald, Masséna, Bernadotte, Davout, Prince Eugène de Beauharnais, Rapp, Thiébault, Jomini) und insofern wenig ergiebig, als diese kaiserlichen Offiziere wenig Kontakt zu Larrey hatten und von ihm nicht behandelt worden waren. 
Dagegen ist man erstaunt, in Marmonts neunbändigem Memoirenwerk keinerlei Hinweise auf Larrey zu finden, wurde er doch von diesem beim Auftreten der Pest in Alexandrien beraten und tatkräftig unterstützt. Auch ergäbe sich bei anderen Gelegenheiten Veranlassung, auf die Leistungen Larreys einzugehen. Allerdings wurde nicht nur Larreys Name stillschweigend übergangen; andere Waffengefährten teilen dasselbe Los (Maréchal Duc de Raguse, Mémoires du Duc de Raguse de 1792 à 1841, imprimés sur le manuscrit original de l'auteur, Paris: Perrotin, Libraire-Editeur 1857).

41 «... sa petite taille» in den Memoiren von Billon (zitiert in Soubiran, Anm. 2).

${ }^{42}$ In den Memoiren (siehe Anm. 2) berichtet Larrey, er hätte sich nach dem Schneiden seiner Haare in mancher Hinsicht nicht wohl gefühlt und die vollständige Integrität seiner physischen und moralischen Funktionen erst zurückgewonnen, wenn die Haare ihre normale Länge wieder erreichten. Um vor seine Majestät Ludwig XVIII. zu treten, ließ er sich die Haare schneiden; doch war der Vorgang prompt gefolgt von Unwohlsein und heftigen Kopfschmerzen. (Er sagt dies in einem Brief an seine Tochter Isaure, zitiert bei Richardson, Anm. 3.)

${ }^{43}$ Ackerknecht, Erwin H., Die Geschichte der Stellung des Chirurgen in der Gesellschaft. In: Helvetica chirurgica Acta 43 (1976).

${ }^{44}$ Ackerknecht, Erwin H., und Fischer-Homberger, Esther, Five made it - One not. The Rise of Medical Craftsmen to Academic Status during the 19th Century. In: Clio Medica 12 (1977), No. 4, S. 255-267.

${ }^{45}$ Stendhal, Aus Napoleons Leben (deutsche Übertragung von «Vie de Napoléon»), München: O.C. Recht 1953.

XIV.Kap. Bonaparte und die Korruption in der Armee.

XVII. Kap. (Rückkehr nach Mailand.) Die Armeelieferanten.

${ }^{46}$ Sabatier-Dupuytren, De la Médecine opératoire, avec des additions et des notes par M.L.-J.Sanson et L.-J.Begin, Paris: Béchet jeune, Librairie de la Faculté de Médecine de Paris, Nouvelle Edition 1832.

Diese Ausgabe des Werkes von Sabatier geht auf die erste Ausgabe vor 36 Jahren zurück, wie im Vorwort vermerkt ist, voller Achtung für den Autor und in Verehrung seines Andenkens. Nicht von ihm selber stammende Textteile sind in Klammern gesetzt. Die nachträglich beigefügten Prolégomènes sind als Zusätze gekennzeichnet.

Im Band IV, Section septième, Paragraphe II, findet sich eine übersichtliche Darstellung aller Amputations- und Exartikulationstechniken. Der jeweils vorangestellte kurze historische Rückblick enthält gründliche Überlegungen zur Entwicklung der einzelnen Eingriffe, wirkt ausgewogen und ist weniger persönlich gefärbt als bei Richerand (siehe Anm. 9). In Klammern gesetzt, also nicht mehr von Sabatier selber, folgen die neuesten Ansichten und operativen Techniken. So erläutert der Autor beispielsweise das frühere Vorgehen Larreys bei der Schulterexartikulation mit Bildung eines äußeren und eines inneren Lappens; in Klammern wird dann die spätere Technik Larreys mit hinterer und vorderer Lappenbildung wiedergegeben (wie sie auch in den Memoiren beschrieben ist). Ganz allgemein ist Sabatier in seiner Indikationsstellung strenger als Larrey. Sabatiers Darlegungen folgen keinerlei Kommentare oder neuere Gesichtspunkte der späteren Bearbeiter des Werkes, so daß sie also in deren Augen, über dreißig Jahre später, noch ihre Gültigkeit beanspruchen durften. Allerdings wird nicht zwischen den einzelnen Veranlassungen zur Amputation oder Exartikulation unterschieden. Während Larrey 
vornehmlich durch seine kriegschirurgischen Erfahrungen geprägt war, sieht Sabatier in der Absetzung einer Gliedmasse «la dernière ressource», die nur praktiziert werden darf, wenn alle Mittel ausgeschöpft sind. Bei der Aufzählung der verschiedenen Indikationen ergeben sich jedoch keine bedeutenden qualitativen Abweichungen gegenüber der Lehrmeinung Larreys.

${ }^{47}$ von Roos, Heinrich, Mit Napoleon in Rußland, Stuttgart: Verlag von Robert Lutz o. J. Ausgabe der Memoirenbibliothek der «Denkwürdigkeiten aus dem Kriege des Jahres $1812 »$ des württembergischen Oberarztes Heinrich von Roos.

Recht aufschlußreich für seine Einstellung mag folgende Passage sein (S. 66): «... sahen wir links der Straße eine grasige Vertiefung, in der viele Arme und Beine und auch Leichen lagen; wir schlossen daraus, daß Larrey mit seinen Kollegen und Ambulanzen gestern hier gearbeitet habe.» Weiter unten (S. 217) spricht er von seinem hervorragenden Bistouri; unter anderm habe er damit «viele brandig gewordene Teile abgenommen ». Also keine primären oder postprimären Amputationen!

Andererseits ist von Roos auch nicht bereit, zu huldigen «dem Arzt, der sich so ernste und wichtige Gesichtszüge angewöhnt hat, daß die Menschen die Unsterblichkeit seiner Patienten in denselben lesen müssen, und der nur Rezepte schreiben kann» (S. 265).

${ }^{48}$ Soubiran, André, Napoléon et un million de morts, Paris: Kent-Segep 1969.

Mit der Wiedergabe von siebzehn bisher nicht edierten Briefen Jean-Baptiste Turiots an den General, den Konsul und den Kaiser Napoleon Bonaparte aus dem Zeitraum von 1796 bis 1814. Turiot war chirurgien aide-major (Bataillonswundarzt) und schließlich auch der Ambulanz der Garde und damit Larrey zugeteilt.

49 d'Eschevannes, Carlos, La vie d'Ambroise Paré, Paris: Librairie Gallimard 1930.

${ }^{50}$ Ackerknecht, Erwin H., Ambroise Paré. In: Image Roche, Nr. 32, 1969, S. 26-32. Amputation im gesunden, nicht abgestorbenen Teil des Gliedes, im Gegensatz zu vielen seiner Kollegen.

${ }^{51}$ Stanhope, Philip Henry, Fifth Earl St., Notes of Conversations with the Duke of Wellington 1831-1851, London: Oxford University Press 1938.

"... he (Wellington) told me that he had been at the hospital at Brussels after the battle of Waterloo, and had seen limbs amputated from several of the French wounded prisoners, but such was their enthusiastic devotion to Napoleon, that even during the operation and admidst their sufferings, they called out 'Vive l'Empereur!'".

${ }^{52}$ Leriche, René, Am Ende meines Lebens, Bern und Stuttgart: Verlag Hans Huber 1957. Im Nachwort von Prof.Dr.med. Hans Debrunner findet sich der Hinweis auf die Vorlesung am Collège de France über das Schmerzproblem.

${ }^{53}$ Blond, Georges, La Beauté et la Gloire. Nelson et Emma Hamilton, Paris : Editions Robert Laffont 1976.

Als behandelnder Chirurg auf der Theseus wird M.Ronicet, exilierter französischer Royalist, genannt. Er konnte die Ansichten Larreys und seiner Kollegen über die Exartikulation im Schultergelenk anstelle der Oberarmamputation nicht kennen und blieb der alten Schule und den Ansichten seiner englischen Berufskollegen treu.

Die rechtsseitige Oberarmamputation wurde am 25.7.1797 vorgenommen. Im Anschlu $\beta$ an den Eingriff litt Nelson an heftigsten Stumpf beschwerden bis zum 30.11.1797, als endlich die letzte Ligatur fiel.

Gegen die Behauptung, Nelson hätte Zeit seines weiteren Lebens stets unter starken 
Beschwerden im Stumpf gelitten, spricht, daß er ihn sehr lebhaft hin und her bewegte, wenn er angespannt oder aufgeregt war, und damit seinen an der Manschette angehängten Uniformärmel in Schwingungen und Flattern versetzte.

${ }^{54}$ Warner, Oliver, The Battle of The Nile, London: B.T.Batsford LTD 1960.

Die Abbildung 35 "Nelson wounded at The Nile. From a portrait by an unknown artist which he gave to Lady Parker" zeigt sehr schön den eher langen Stumpf des rechten Oberarmes.

Weitere Nelson-Biographien, so von C.S. Forester, Arthur Bryant, Carola Oman, Oliver Warner und Robert Southey, sind weniger ausführlich über diese Verletzung, den Eingriff und den anschließenden Verlauf.

${ }^{55}$ In Dominique Jean Larreys Chirurgischer Klinik (siehe Anm. 1), Band III, S. 427 f., findet sich das Kapitel «Von den Amputationen». Viele Beobachtungen und Fallbesprechungen sind bereits in den Memoiren angeführt (1812-1817), jedoch nun zum Teil weiterverfolgt und durch den späteren Verlauf ergänzt. Große Abweichungen von den Lehrmeinungen der Kriegszeit während der Revolution und des Kaiserreiches sind für die nachfolgenden Jahre nicht zu verzeichnen. Die Hauptsätze sind noch deutlicher ausgearbeitet und eindringlicher vorgetragen; einzig die primäre Naht geeigneter Stümpfe wird jetzt in Ausnahmefällen gestattet. Die Verbandtechnik hat kleine Änderungen erfahren, ebenso die Drainage bei Exartikulation.

Zwischen der "primitiven» und der «consecutiven» Amputation unterscheidet der Autor in diesem Werk deutlicher. Die letztere kommt am «lieu d'élection» in Frage bei trockener Gangrän (vor allem bei Gangraena senilis), bei Brand durch Erfrierung, bei Nachresektionen und Stumpfkorrekturen (wo man aber immer sehr zurückhaltend sein soll), bei Knochennekrose, Spina ventosa, Osteosarkom und beim Vorliegen karzinomatöser Tuberkeln.

Zu den Exartikulationsoperationen: Im Schultergelenk ist die Blutung aus der Arteria circumflexa externa problematisch; doch läßt sich dieses Gefäß bei Befolgung von Larreys operationstechnischen Weisungen unter Sicht behalten und ligieren. Von 111 Fällen sind 97 Erfolge vermeldet. Die Ellbogenexartikulation soll zugunsten des nächsttieferen oder nächsthöheren Niveaus aufgegeben werden. Das gilt auch für das Knie, wo die "Amputation in der unteren Demarcationslinie des Kniegelenkes» oder die "Amputation in den Condylen der Tibia», kurz die hohe Unterschenkelamputation unter Schonung von Kapsel, Seitenbandapparat und Ligamentum patellae vorzuziehen ist. Betreffend die Hüftexartikulation stellt sich Larrey in Gegensatz zu Guthrie, indem er die vorläufige Unterbindung des Femoralgefäßes von einem zu Beginn der Operation durchgeführten Längsschnitt aus verficht.

Bei den Amputationen kann die Absetzung im Oberarm nur diskutiert werden, wenn der Stumpf lang belassen werden kann. Sonst ist der Schulterexartikulation der Vorzug zu geben. Die Vorderarmamputation soll ellbogennahe erfolgen, aus ähnlichen Überlegungen heraus, wie auch ein hohes Amputationsniveau am Unterschenkel zu wählen ist. Die Oberschenkelamputation hinwiederum soll möglichst distal erfolgen, ebenfalls in den meisten Fällen ohne Lappenbildung. Wenn schon Lappen am Ober- und Unterschenkel geformt werden sollen, dann erst im Anschluß an die Absetzung der Extremität durch mehretagigen Zirkelschnitt und mit vorderer und hinterer perpendikulärer Inzision zur Bildung von «Seitenlappen». Partialamputationen des Fußes sind nur ange- 
zeigt, wenn sie möglichst weit distal im Vorder- oder Mittelfuß erfolgen können. Die Narbe soll dann dorsalseitig zu liegen kommen, unter Heraufschlagen eines längeren plantaren Hautlappens.

56 Diese Zeiten sind, wenngleich nicht unter denselben Bedingungen, nachvollziehbar, und zwar beim probatorischen Anlegen von Zirkelschnitten zur definitiven Bestimmung der Amputationshöhe bei Gefäßpatienten. Wäre man wirklich äußerst geschickt und sehr geübt, könnte man beispielsweise eine Unterschenkelamputation in ähnlich kurzer Zeit vornehmen. Für eine Hüftexartikulation brauchte man auch bei idealen Voraussetzungen länger. Diese Operationszeiten Larreys sind phantastisch, und doch glauben wir sie ihm und trauen sie seiner operativen Meisterschaft zu.

${ }^{57}$ Vgl. beispielsweise Bonham-Carter, Victor, Surgeon in the Crimea. The Experiences of George Lawson recorded in Letters to his Family 1854-1855. London: Military Book Society 1968.

${ }^{58}$ Ackerknecht, Erwin H., Medicine at the Paris Hospital 1794-1848, Baltimore: The Johns Hopkins Press 1967.

\section{Summary}

Dominique Jean Larrey's name as an outstanding surgeon, especially as one of the greatest military surgeons, is known for many good reasons. He developed and published operative techniques which saved and healed so many war-victims.

The details of his successful operative technique are presented. The fact he was not only an excellent surgeon but also a great physician is obvious in many references and deserves further examination.

Dr.med.Beat Rüttimann

Orthopädische Universitätsklinik Balgrist

Forchstraße 340

8008 Zürich 